$03,10,19$

\title{
Влияние фокусировки фононов на теплопроводность гетероструктур GaAs/AIGaAs при низких температурах
}

\author{
() И.И. Кулеев \\ Институт ффизики металлов УрО РАН, \\ Екатеринбург, Россия \\ E-mail: kuleyevll@imp.uran.ru
}

(Поступила в Редакцию 1 октября 2018 г.)

Исследовано влияние анизотропии упругих свойств на теплопроводность гетероструктур GaAs/AlGaAs при низких температурах. Проанализировано влияние фокусировки фононов на анизотропию теплопроводности. Определены параметры зеркальности отражения фононов от границ гетероструктур, характеризующие тепловой поток в режиме кнудсеновского течения фононного газа. Рассчитаны угловые зависимости длин свободного пробега фононов различных поляризаций, определяющих анизотропию теплопроводность гетероструктур с ориентациями плоскостей $\{100\}$ и $\{110\}$.

Работа выполнена по плану РАН в рамках темы „Спин“ № АААА-А18-118020290104-2, по проектам программ Президиума РАН № 32-1.1.3.5 и УрО РАН № 18-10-2-37.

DOI: 10.21883/FTT.2019.03.47231.271

\section{1. Введение}

Явления электронного и фононного транспорта в гетероструктурах GaAs/AlGaAs исследовались в большом количестве работ [1-9]. Однако эффекты, обусловленные фокусировкой фононов, при этом не учитывались: не указывались направления теплового потока и ориентаций боковых граней гетероструктур. Исключение составляет работа [1], в которой измерены теплопроводность и термоэдс электрон-фононного увлечения в трех гетероструктурах $\mathrm{GaAs} / \mathrm{AlGaAs}$, имеющих различные ориентации плоскостей и направления потока тепла. Это позволяет проанализировать роль фокусировки фононов в теплопроводности и термоэдс увлечения. Ранее при интерпретации экспериментальных данных по теплопроводности гетероструктур использовалась модель изотропной среды [2-9], и длины свободного пробега фононов оценивались из выражений, полученных для фононного транспорта в пластинках в одномодовом приближении [8,9]. В работе [3] показано, что полученные оценки в режиме граничного рассеяния отличаются от результатов эксперимента в гетероструктурах GaAs/AlGaAs в $2-2.5$ раза.

Известно, что анизотропия упругой энергии кубических кристаллов приводит к неколлинеарности фазовой и групповой скоростей фононов и их фокусировке [10-13]. Экспериментальные исследования [11] показали, что при достаточно низких температурах, когда длина свободного пробега фононов превышает поперечный размер образца, фокусировка фононов приводит к зависимости анизотропии теплопроводности. Для образцов Si с квадратным сечением она становится зависящей от направления теплового потока, а для образцов с прямоугольным сечением - в значительной степени от ориентации широкой грани образца. В работах [14-16] решена задача о диффузном рассеянии фононов на границах образцов конечной длины с круглым, квадратным и прямоугольным сечениями и найдены аналитические выражения для времен релаксации фононов. Использование этих решений позволило адекватно описать экспериментальные данные по теплопроводности кристаллов кремния [7,17] во всем диапазоне температур для различных направлений градиента температуры и ориентаций боковых граней образцов.

В настоящей работе эти результаты будут использованы для анализа анизотропии теплопроводности гетероструктур $\mathrm{GaAs} / \mathrm{AlGaAs}$. Особенности термоэлектрических явлений в гетероструктурах обусловлены взаимодействием квазидвумерного электронного газа с трехмерными фононами подложки GaAs, имеющей макроскопические размеры [1-9]. Для исследования влияния фокусировки на термоэдс увлечения необходимо, прежде всего, проанализировать механизмы релаксации фононов, определяющие тепловой поток в низкотемпературной области. В работе [1] теплопроводность и термоэдс гетероструктур $\mathrm{GaAs} / \mathrm{AlGaAs}$ измерялась при температурах $0.2-1 \mathrm{~K}$. Было показано, что в этом температурном интервале теплопроводность следовала зависимости $T^{3}$, что соответствует доминирующей роли граничного рассеяния фононов. Поэтому в настоящей работе мы ограничимся анализом анизотропии теплопроводности, и определим релаксационные параметры, характеризующих тепловой поток в гетероструктурах в режиме кнудсеновского течения фононного газа.

\section{2. Фокусировка фононов и анизотропия длин свободного пробега фононов в кристаллах GaAs}

При низких температурах для анализа влияния фокусировки на распространение и релаксацию фононов 
Упругие модули второго порядка $c_{i j}\left(10^{12} \mathrm{dyn} / \mathrm{cm}^{2}\right)$, плотность $\rho\left(\mathrm{g} / \mathrm{cm}^{3}\right)$, углы $\theta_{1}^{t 2\{100\}}, \theta_{2}^{t 2\{100\}}, \theta_{3}^{t 2\{100\}}, \theta_{4}^{t 2\{100\}}$ для кристаллов GaAs [22] и $\mathrm{Si}$

\begin{tabular}{c|c|c|c|c|c|c|c|c|c}
\hline Соединение & $c_{11}$ & $c_{12}$ & $c_{44}$ & $k-1$ & $\rho$ & $\theta_{1}^{t 2\{100\}}$ & $\theta_{2}^{t 2\{100\}}$ & $\theta_{3}^{t 2\{100\}}$ & $\theta_{4}^{t 2\{100\}}$ \\
\hline $\mathrm{GaAs}$ & 1.226 & 0.571 & 0.60 & 0.87 & 5.36 & $32.4^{\circ}$ & $13.7^{\circ}$ & $12.5^{\circ}$ & $32.4^{\circ}$ \\
$\mathrm{Si}$ & 1.677 & 0.65 & 0.804 & 0.67 & 2.33 & $23.6^{\circ}$ & $11.9^{\circ}$ & $6.8^{\circ}$ & $28.6^{\circ}$
\end{tabular}

в монокристаллах GaAs воспользуемся моделью анизотропного континуума [18-19]. В этой модели спектр фононов с поляризацией $\lambda$ при $q \ll q_{D}$ может быть представлен в виде $\omega_{q}^{\lambda}=S^{\lambda}(\theta, \varphi) q$, где фазовая скорость $S^{\lambda}(\theta, \varphi)$, зависящая от от угловых переменных $\theta$ и $\varphi$ вектора q, определяет анизотропию спектра (см. подробнее [20]). Индекс поляризации фононов $L$ соответствует продольным фононам, а $t_{1}$ и $t_{2}-$ соответственно, „быстрой“ (верхней) и „медленной“ (нижней) поперечным колебательным модам. Ранее в работах $[14,16]$ проведены детальные расчеты теплопроводности и длин свободного пробега фононов в кристаллах $\mathrm{Si}$ и показано, что при низких температурах они согласуются с результатами [11] в пределах погрешности эксперимента. Поэтому при анализе анизотропии длин свободного пробега фононов в кристаллах GaAs мы будем рассматривать кристаллы $\mathrm{Si}$ как систему сравнения. Следует отметить, что величины упругих модулей $c_{i j}$ в кристаллах GaAs несколько меньше, чем в $\mathrm{Si}$, а их плотность в 2.3 раза больше, чем в $\mathrm{Si}$ (см. таблицу). Значения модулей упругости второго порядка для GaAs при комнатной температуре были измерены в работах [21]. Позднее в работе [22] были проведены измерения при температуре $77 \mathrm{~K}$ и при анализе кинетических явления в кристаллах GaAs они были экстраполированы на $T=0 \mathrm{~K}$. Поскольку в [1] теплопроводность измерялись в интервале от 0.2 до $1 \mathrm{~K}$ то мы будем использовать значения, экстраполированные на $T=0 \mathrm{~K}$ (см. таблицу). В работе [20] было показано, что анизотропия спектра фононов определяется параметром $k-1 \quad\left(k-1=\left(c_{12}+2 c_{44}-c_{11}\right) /\left(c_{11}-c_{44}\right)\right.$, $c_{i j}$ - упругие модули второго порядка). При $T=0 \mathrm{~K}$

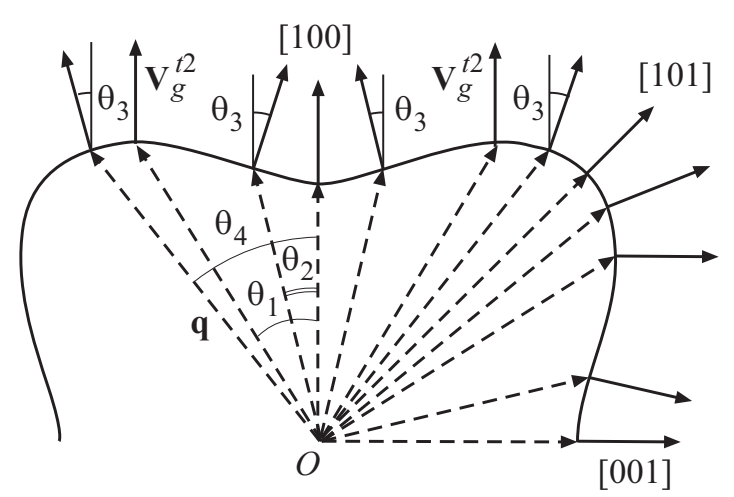

Рис. 1. Сечение изоэнергетической поверхности плоскостью $X Z$ в кристалле GaAs для медленной поперечной моды. Стрелками изображены волновые векторы внутри поверхности и соответствующие им групповые скорости фононов вне ее. параметр $k-1$ в кристаллах GaAs оказался равным 0.87 , что заметно больше, чем в $\mathrm{Si}$ (см. таблицу).

Для кристаллов $\mathrm{GaAs}$ и $\mathrm{Si}$ наиболее анизотропной является медленная поперечная мода $t_{2}$. Проиллюстрируем фокусировку фононов на примере этой моды. Построим для нее изоэнергетическую поверхность (см. рис. 1). Групповая скорость фонона, определяющая направление переноса энергии, перпендикулярна этой поверхности. Для произвольного направления волнового вектора она может быть представлена в виде [22]

$$
\begin{gathered}
\mathbf{V}_{g}^{\lambda}(\theta, \varphi)=S^{\lambda}(\theta, \varphi) \overline{\mathbf{V}}_{g}^{\lambda}(\theta, \varphi), \\
\overline{\mathbf{V}}_{g}^{\lambda}(\theta, \varphi)=\mathbf{n}+S_{\theta}^{\lambda} \mathbf{e}_{\theta}+S_{\varphi}^{\lambda} \mathbf{e}_{\varphi}, \\
S_{\theta}^{\lambda}(\theta, \varphi)=\left(\frac{1}{S^{\lambda}}\right) \frac{\partial S^{\lambda}}{\partial \theta}, \quad S_{\varphi}^{\lambda}(\theta, \varphi)=\frac{1}{\sin \theta}\left(\frac{1}{S^{\lambda}}\right) \frac{\partial S^{\lambda}}{\partial \varphi} .
\end{gathered}
$$

Здесь

$$
\begin{gathered}
\mathbf{e}_{\theta}=(\cos \theta \cos \varphi, \cos \theta \sin \varphi,-\sin \theta), \\
\mathbf{e}_{\varphi}=(-\sin \varphi, \cos \varphi, 0),
\end{gathered}
$$

a $\mathbf{n}=\mathbf{q} / q=(\sin \theta \cos \varphi, \sin \theta, \sin \varphi, \cos \theta)-$ единичный волновой вектор фонона. Вектора $\mathbf{n}, \mathbf{e}_{\theta}$ и $\mathbf{e}_{\varphi}$ образуют взаимно ортогональную тройку единичных векторов. Из рис. 1 видно, что фокусировка фононов характеризуется углами: $\theta_{1}-\theta_{4}$ (см. подробнее $\left.[17,23,24]\right)$.

В изотропной среде направление распространения фонона и его волнового вектора совпадают, поэтому фононы, распространяющиеся в изотропной среде в секторе $-\theta_{4} \leq \theta \leq \theta_{4}$, в кристаллах GaAs будут распространяться в существенно меньшем секторе $-\theta_{3} \leq \theta \leq \theta_{3}$ (см. рис. 1). Таким образом, плотность состояний для моды $t_{2}$ в направлениях фокусировки близких к [100] существенно возрастает по сравнению с изотропной средой. Оценим влияние фокусировки на плотность фононных состояний (ПФС), аналогично тому, как это сделано в [23] (см. также [17]). Как видно из таблицы, большие значения параметра анизотропии $k-1$ приводят к тому, что в кристаллах GaAs углы $\theta_{3}$ и $\theta_{4}$ в 1.84 и 1.13 раза больше, чем в $\mathrm{Si}$. Увеличение значения угла $\theta_{3}$ для GaAs, приводит, во-первых, к увеличению области фокусировки медленной поперечной моды, во-вторых, к уменьшению значения плотности фононных состояний в этой области относительно кристаллов Si. Результаты расчета ПФС моды $t_{2}$ для GaAs показали, что в области фокусировки она превосходит значение в модели изотропной среды в 2.6 раза, а в области дефокусировки ее 


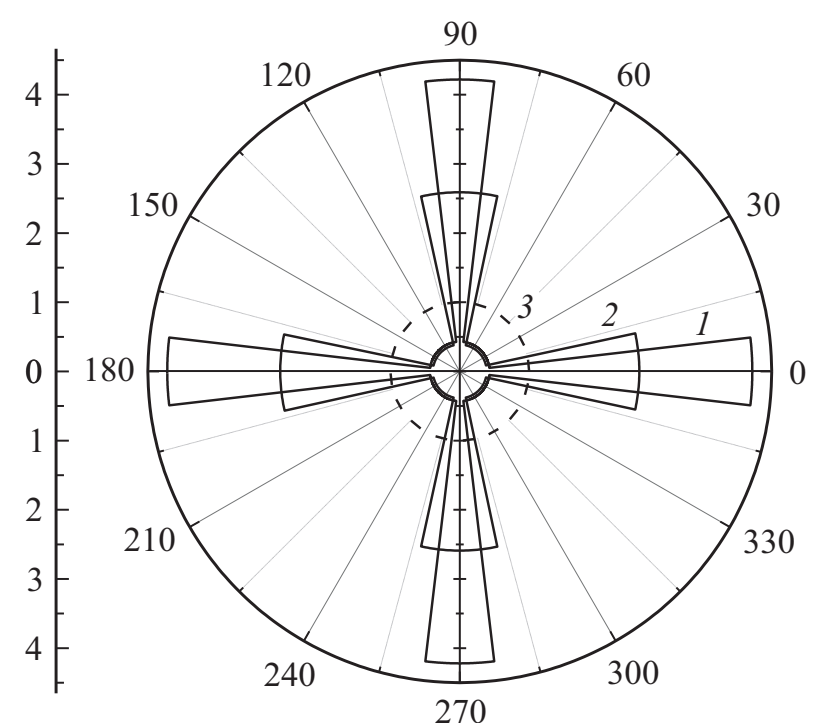

Рис. 2. Угловые зависимости средних плотностей фононных состояний моды $t_{2}$ для волновых векторов вплоскости $\{100\}$ в кристаллах $\mathrm{Si}(1), \mathrm{GaAs}(2)$, изотропная среда кривая (3).

значение в 2.6 раза меньше, чем в модели изотропной (см. рис. 2). В результате ПФС в области фокусировки оказывается в 6.7 раза больше, чем в области дефокусировки. Как видно из рис. 2, в кристаллах $\mathrm{Si}$ это отношение оказывается равным 9.9. Аналогично можно показать, что продольные фононы фокусируются в направлении [111], а быстрая поперечная мода - в направлении [110] (см. [17]).

Проанализируем анизотропию теплопроводности образцов GaAs тех же геометрических размеров, что и в работе [14] для образцов $\mathrm{Si}$, чтобы иметь возможность сравнить результаты с экспериментальными данными: то есть возьмем стержень GaAs с квадратным сечением $D=0.293 \mathrm{~cm}$ и длиной $L=2.9 \mathrm{~cm}$. При температурах, гораздо меньших температуры Дебая $\left(T \ll T_{D}\right)$ представим теплопроводность в виде аддитивной суммы вкладов от всех колебательных ветвей (см. $[15,17,25])$

$$
\begin{gathered}
\kappa_{[I(\psi)]}^{\{J\}}(T)=\frac{1}{3} C_{V}(T) \bar{S} \widetilde{\Lambda}_{[I(\psi)]}^{\{J\}}=\sum_{\lambda} \frac{1}{3} C_{V}^{\lambda} \bar{S} \Lambda_{[I(\psi)]}^{\{J\} \lambda}, \\
C_{V}=\frac{2 \pi^{2} k_{\mathrm{B}}^{4}}{5 \hbar^{3}} \frac{1}{3}\left\langle\left(S^{\lambda}\right)^{-3}\right\rangle, \\
\bar{S}=\sum_{\lambda}\left\langle\left(S^{\lambda}\right)^{-2}\right\rangle / \sum_{\lambda}\left\langle\left(S^{\lambda}\right)^{-3}\right\rangle, \\
\bar{S}^{\lambda}=\left\langle\left(S^{\lambda}\right)^{-2}\right\rangle /\left\langle\left(S^{\lambda}\right)^{-3}\right\rangle, \\
\left\langle\left(S^{\lambda}\right)^{-n}\right\rangle=\frac{1}{4 \pi} \int d \Omega_{q}\left(S_{\lambda}\right)^{-n}, \quad d \Omega_{q}=\sin \theta d \theta d \varphi .
\end{gathered}
$$

Удельные теплоемкости $C_{V}(T)$ и $C_{V}^{\lambda}(T)$, а так же средние скорости фонона $\bar{S}$ и $\bar{S}^{\lambda}$ не зависят от направления потока тепла. Поэтому анизотропия теплопроводности и вкладов в нее от колебательных мод определяются длинами свободного пробега $\Lambda_{[I(\psi)]}^{\{J\}}$ и $\Lambda_{[I(\psi)]}^{\{J\} \lambda}$. Мы рассчитали угловые зависимости длин пробега фононов для случаев, когда тепловой поток вращается в плоскости грани куба $Y Z \quad\{J\}=\{100\}$ или в диагональной плоскости $\{J\}=\{110\}$. Для анизотропии теплопроводности и средних длин пробега в кристаллах $\mathrm{GaAs}$ и $\mathrm{Si}$ мы получили близкие результаты: в кристаллах GaAs $\widetilde{\Lambda}_{[100]}^{\{100\}}: \widetilde{\Lambda}_{[110]}^{\{100\}}: \widetilde{\Lambda}_{[111]}^{\{110\}}=$ $=1.45: 1.07: 1$, в кристаллах $\mathrm{Si} \widetilde{\Lambda}_{[100]}^{\{100\}}: \widetilde{\Lambda}_{[110]}^{\{100\}}: \widetilde{\Lambda}_{[111]}^{\{110\}}=$ $=1.50: 1.08: 1 \quad$ (см. рис. 3$)$. Отметим, что для
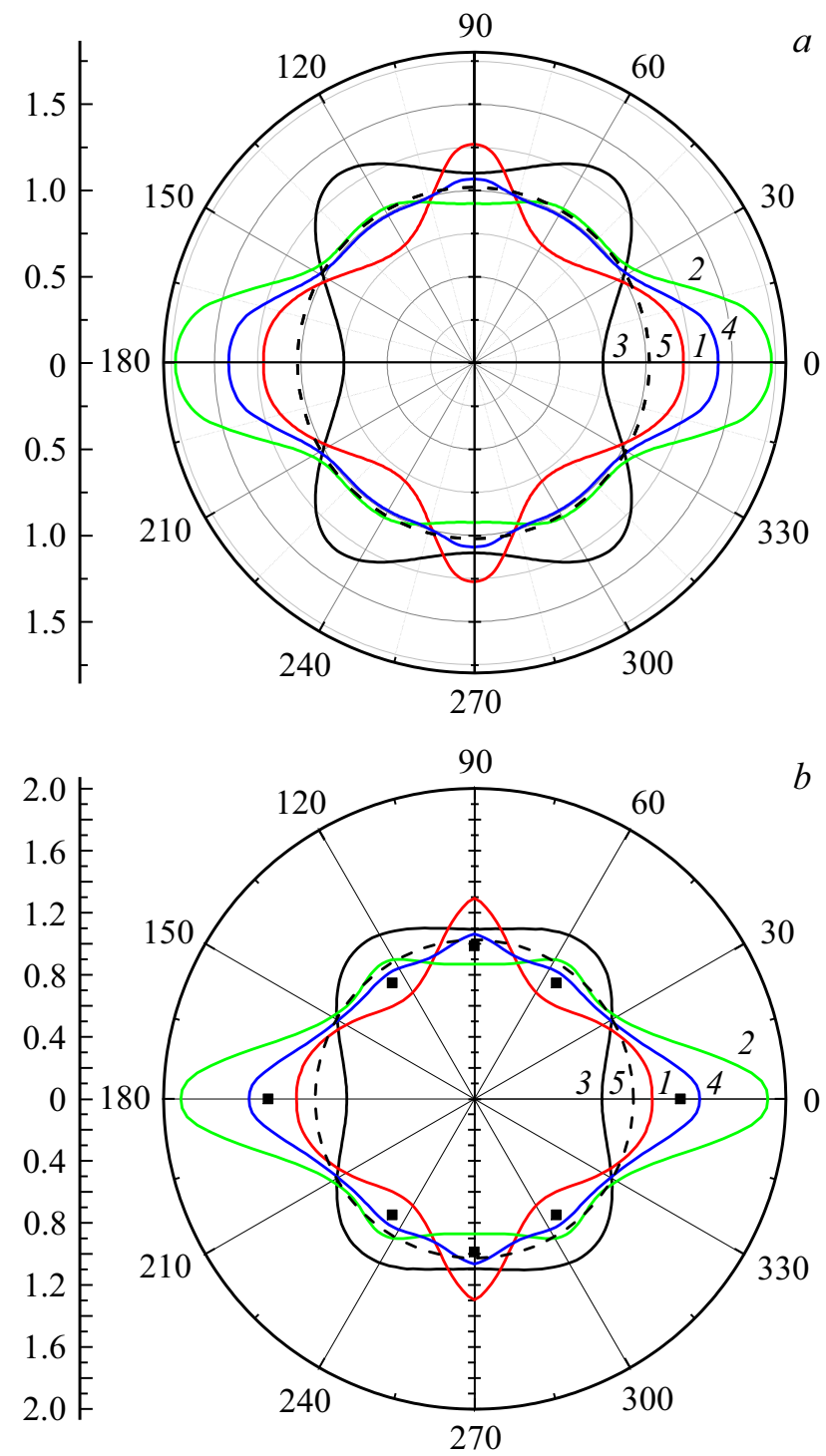

Рис. 3. Угловые зависимости приведенных длин свободного пробега фононов $\widetilde{\Lambda}_{I(\psi)}^{\{J\}}=\Lambda_{I(\psi)}^{\{J\} \lambda} / D$ и $\widetilde{\Lambda}_{I(\psi)}^{\{J\}}=\Lambda_{I(\psi)}^{\{J\}} / D$ в GaAs $(a)$ и $\mathrm{Si}(b)$ для образцов с квадратным сечением $D=0.293 \mathrm{~cm}$ и длиной $L=2.9 \mathrm{~cm}$ в случаях, когда градиент температуры вращается в диагональной плоскости: 1 - быстрая поперечная мода, 2 - медленная поперечная мода, 3 - продольная мода, 4 - средняя длин свободного пробега. Пунктирная 5 - для изотропной среды $\left(\Lambda_{i s o}=1.12 D\right)$. Точки - экспериментальные значения длин пробега для $\mathrm{Si}[11]$. 
кристаллов $\mathrm{Si}$ результаты расчета в симметричных направлениях [14] хорошо согласуются с экспериментальными данными [11]. Как видно из рис. 3, для образцов с квадратным сечением длины свободного пробега фононов для каждой колебательной моды в кристаллах $\mathrm{GaAs}$ и $\mathrm{Si}$ достигают максимальных значений в направлениях их фокусировки, причем в этих направлениях они превосходят длины пробега фононов остальных колебательных мод. Так, например, в кристаллах $\mathrm{GaAs}$ для отношения длин пробега имеем: в направлениях типа [100] $\Lambda_{[100]}^{\{100\} t_{2}}: \Lambda_{[100]}^{\left\{100 t_{1}\right.}: \Lambda_{[100]}^{\{100\} L}=$ $=2.32: 1.67: 1 \quad$ в направлениях типа [111] $\Lambda_{[111]}^{\{110\} L}: \Lambda_{[111]}^{\{110\} t_{2}}: \Lambda_{[111]}^{\{110\} t_{1}}=1.72: 1.32: 1$, а в направлениях типа $[110] \Lambda_{[110]}^{\{100\} t_{1}}: \Lambda_{[110]}^{\{100\} L}: \Lambda_{[110]}^{\{100\} t_{2}}=1.40: 1.25: 1$ (см. рис. 3). Качественно подобные результаты получаются и для кристаллов $\mathrm{Si}$ в в направлениях типа [100] $\Lambda_{[100]}^{\left\{100 t_{2}\right.}: \Lambda_{[100]}^{\{100\} t_{1}}: \Lambda_{[100]}^{\{100\} L}=2.33: 1.46: 1$, в направлениях типа $[111] \Lambda_{[111]}^{\{110\} L}: \Lambda_{[111]}^{\left\{110 t_{2}\right.}: \Lambda_{[111]}^{\{110\} t_{1}}=1.56: 1.35: 1$, а в направлениях типа $[110] \Lambda_{[110]}^{\{100\} t_{1}}: \Lambda_{[110]}^{\{100\} L}: \Lambda_{[110]}^{\left\{100 t_{2}\right.}=$ $=1.50: 1.27: 1$.

Из приведенных выше результатов следует, что анизотропия теплопроводности в кристаллах $\mathrm{GaAs}$, как и в $\mathrm{Si}$, в режиме граничного рассеяния обусловлена, главным образом, медленной поперечной модой, которая фокусируется в направлении [100] и обеспечивает максимум теплопроводности в этом направлении. Ee вклад составляет 63 и 61\% для кристаллов $\mathrm{GaAs}$ и $\mathrm{Si}$ соответственно. Направление [100] соответствует направлению дефокусировки продольных фононов. Их вклад в теплопроводность кристаллов $\mathrm{GaAs}$ и $\mathrm{Si}$ минимален и составляет 7 и 8\% соответственно. В направлениях типа [111] фокусируются продольные фононы, и их вклад в теплопроводность кристаллов $\mathrm{GaAs}$ и $\mathrm{Si}$ возрастает более, чем в 2 раза и составляет 18 и 19\% соответственно. Таким образом, поперечные фононы вносят преобладающий вклад в теплопроводность этих кристаллов.

\section{3. Анизотропия теплопроводности гетероструктур GaAs/AIGaAs с различной ориентацией плоскостей при низких температурах}

В работе [1] были измерены зависимости теплопроводности гетероструктур GaAs/AlGaAs от температуры в интервале от 0.2 до $1 \mathrm{~K}$. Подложки GaAs имели прямоугольную форму с геометрическими размерами: для первого образца с размерами $9.5 \times 4 \times 0.4 \mathrm{~mm}$, ориентацией плоскости гетероструктуры 100 и направлением потока тепла [100], а для второго образца с размерами $9.5 \times 4.5 \times 0.4 \mathrm{~mm}$, ориентацией плоскости гетероструктуры $\{110\}$ и направлением теплового потока [110]. Анализ теплопроводности в трехмодовой модели Каллавея [14-16] показал, что в интервале от 0.2 до

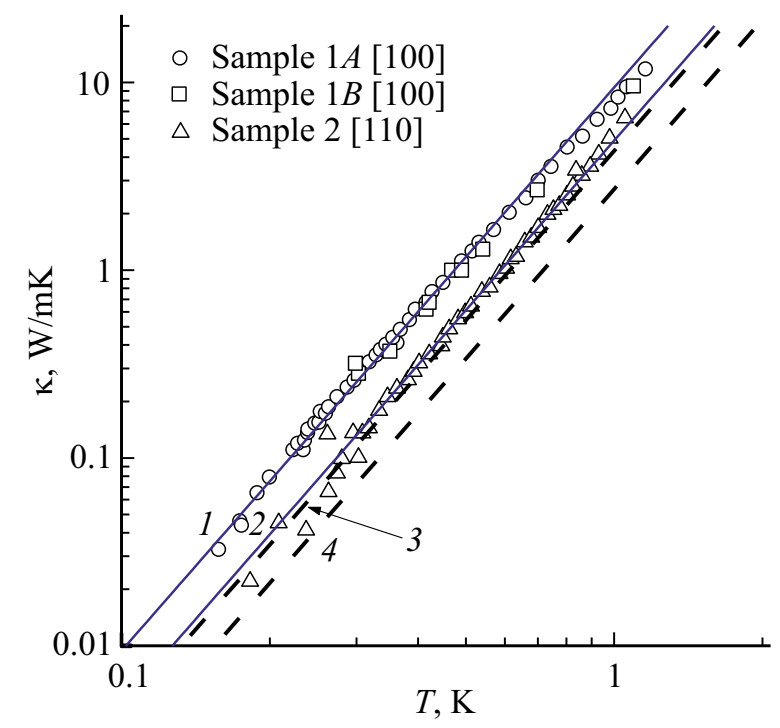

Рис. 4. Температурные зависимости теплопроводности гетероструктур GaAs с ориентациями плоскостей $\{100\}$ и $\{110\}$ : $1-\kappa^{[100]}=9.4 T^{3}, 2-\kappa^{[110]}=4.9 T^{3}, 3$ и $4-$ рассчитанные при полностью диффузном рассеянии для направления [100] и [110]. Символы - экспериментальные значения теплопроводности [1].

$1 \mathrm{~K}$ ангармонические процессы рассеяния выморожены, вклад в теплосопротивление от рассеяния на изотопическом беспорядке мало - оно составляет менее $0.2 \%$. Согласно [1] в интервале $(0.2-0.8) \mathrm{K}$ теплопроводность следовала зависимости $T^{3}$, что соответствует преобладающей роли граничного рассеяния фононов. При более высоких температурах $(0.8-1) \mathrm{K}$ степень температурной зависимости уменьшается, и экспериментальные зависимости для гетероструктур с различными ориентациями начинают сближаться (см. рис. 4). Таким образом, в интервале от 0.2 до $0.8 \mathrm{~K}$ в гетероструктурах GaAs реализуется случай кнудсеновского течения фононного газа. Оценки показывают, что в этом режиме теплопроводность для ориентации плоскости $\{100\}$ оказывается в 1.9 раза больше, чем для ориентации $\{110\}$. В этом режиме единственным подгоночным параметром теории является параметр зеркальности. В отличие от объемных образцов, где можно обеспечить диффузный характер рассеяние фононов на границах путем обработки поверхности образца наждаком (см. [11]), для нано и гетероструктур такая обработка невозможна. Поэтому мы учтем частичную зеркальность рассеяния фононов обычным образом [26-27]:

$$
\begin{gathered}
\kappa_{[I(\psi)]}^{\{J\}}(T)=C_{V}(T) \bar{S} \Lambda_{[I(\psi)]}^{\{J\}}(T) / 3=\sum_{\lambda} C_{V}^{\lambda}(T) \bar{S}^{\lambda} \Lambda_{[I(\psi)]}^{\{J\} \lambda} / 3 \\
\Lambda_{[I(\psi)]}^{\{J\}}=\Lambda_{[I(\psi)]}^{\{J\}}[(1+P) /(1-P)],
\end{gathered}
$$

где $P$ - фактор зеркальности, а $\Lambda_{[I(\psi)]}^{\{J\}}$ - длина свободного пробега фононов при диффузном отражении от границ гетероструктуры, ориентационные параметры $[I]$ 
и $\{J\}$ характеризуют зависимости теплопроводности и длин пробега от направления теплового потока $[I]$ и ориентации плоскости гетероструктуры $\{J\}$. Как видно из рис. 4 , при учете факторов зеркальности $P_{1}=0.37$ для первой гетероструктуры и $P_{2}=0.29$ - для второй результаты расчета теплопроводности в низкотемпературной области хорошо согласуются с экспериментальными данными [1]. Поскольку параметры зеркальности для гетероструктур имеют различные значения, то отношение величин их теплопроводности зависит не только от отношений длин свободного пробега фононов при диффузном отражении от границ, но и значений параметров зеркальности

$$
\begin{aligned}
\kappa_{[100]}^{\{100\}}(T) / \kappa_{[110]}^{\{110\}}(T)=\widetilde{\Lambda}_{[100]}^{\{100\}} / \widetilde{\Lambda}_{[110]}^{\{10\}} & \\
= & \frac{1+P_{1}}{\left(1-P_{1}\right)} \Lambda_{[100]}^{\{100\}} /\left\{\frac{1+P_{2}}{\left(1-P_{2}\right)} \Lambda_{[110]}^{\{110\}}\right\} .
\end{aligned}
$$

Для полученных значений отношения параметров зеркальности дает коэффициент 1.2, то есть за счет различия параметров зеркальности гетероструктур на 20увеличивается различие их теплопроводности. При полностью диффузном отражении фононов от границ гетероструктур с ориентацией плоскостей $\{100\}$ и $\{110\}$, их теплопроводности отличались бы 1.6 , а не в 1.9 раза, как имело место в эксперименте [1].

Проанализируем угловые зависимости теплопроводности гетероструктур аналогично тому, как это сделано в [25]. Рассмотрим вращение теплового потока в плоскости гетероструктуры для двух случаев: (1) плоскость гетероструктуры имеет ориентацию $\{J\}=\{100\}$, (2) плоскость гетероструктуры совпадает с диагональной плоскостью $\{J\}=\{110\}$. Ориентационные параметры $[I(\psi)]$ и $\{J\}$ для произвольного направления теплового потока относительно осей кристалла могут быть определены через компоненты групповой скорости, параллельные и перпендикулярные тепловому потоку. Определим систему координат с осью „3“ вдоль направления теплового потока. Ось „1“ (ось вращения) направим перпендикулярно плоскости гетероструктуры, она определяет ориентацию плоскости $\{J\}$. Ось „,“ направим перпендикулярно двум узким боковым граням пленки. Тогда компоненты групповой скорости фононов для рассматриваемых случаев могут быть представлены в следующем виде [25]:

$$
\begin{gathered}
V_{g 3}^{\lambda}=-V_{g y}^{\lambda} \sin \psi+V_{g z}^{\lambda} \cos \psi, \\
V_{g 2}^{\lambda}=-V_{g y}^{\lambda} \cos \psi+V_{g z}^{\lambda} \sin \psi, \quad V_{g 1}^{\lambda}=V_{g x}^{\lambda}, \\
V_{g 3}^{\lambda}=\left(-V_{g x}^{\lambda}+V_{g y}^{\lambda}\right) \sin \psi / \sqrt{2}+V_{g z}^{\lambda} \cos \psi, \\
V_{g 2}^{\lambda}=\left(-V_{g x}^{\lambda}+V_{g y}^{\lambda}\right) \cos \psi / \sqrt{2}-V_{g z}^{\lambda} \sin \psi, \\
V_{g 1}^{\lambda}=\left(V_{g x}^{\lambda}+V_{g y}^{\lambda}\right) / \sqrt{2} .
\end{gathered}
$$

Зависимость направления теплового потока от угла $\psi$ определяется компонентой групповой скорости $V_{g 3}^{\lambda}$. Проекция групповой скорости $V_{g 1}^{\lambda}$ не зависит от угла $\psi$,
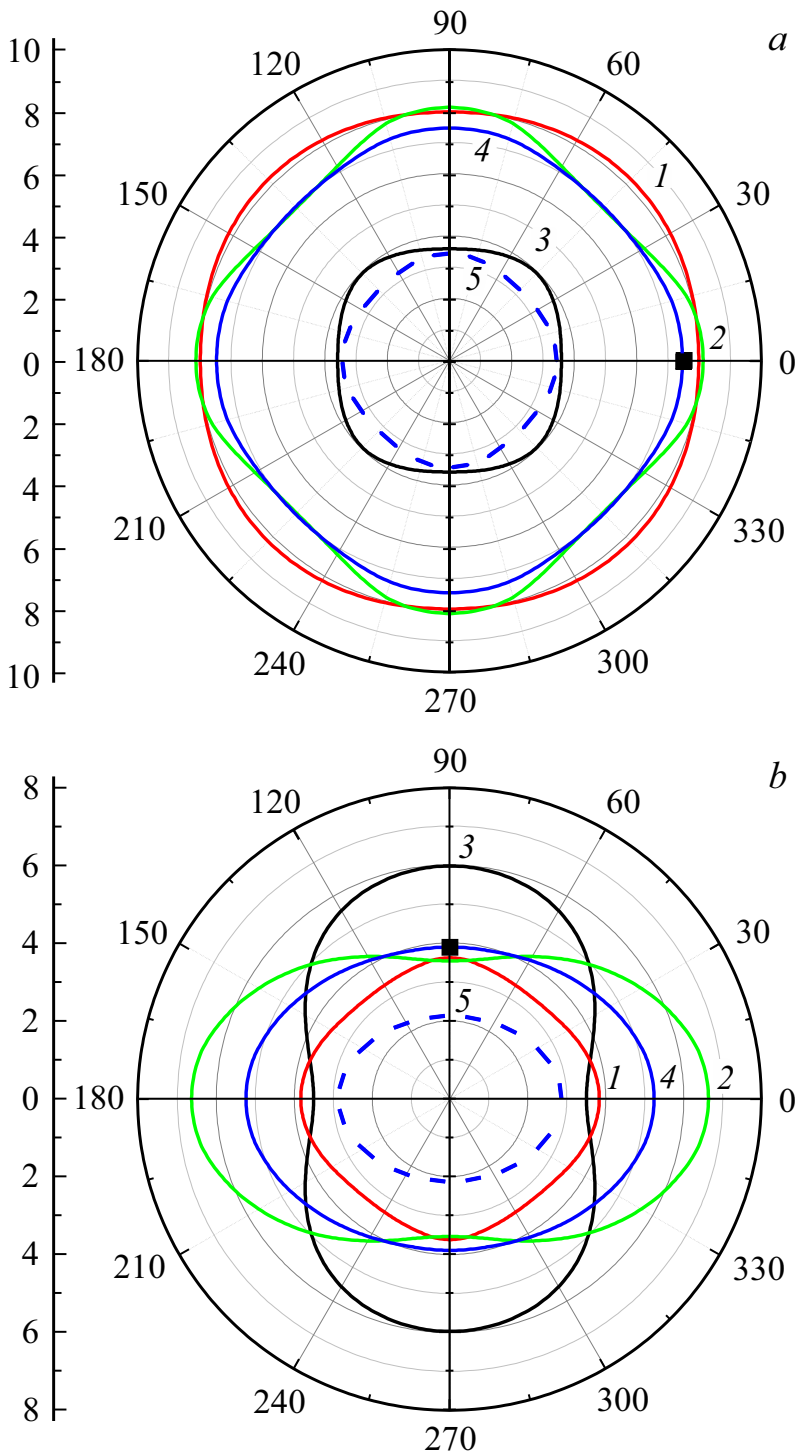

Рис. 5. Угловые зависимости приведенных длин свободного пробега фононов $\widetilde{\Lambda}_{I(\psi)}^{\{J\} \lambda}=\Lambda_{I(\psi)}^{\{J\} \lambda} / D$ и $\widetilde{\Lambda}_{I(\psi)}^{\{J\}}=\Lambda_{I(\psi)}^{\{J\}} / D$ для гетероструктур GaAs c ориентациями плоскостей $(a)-\{100\}$ и $(b)-\{110\} .1-$ для быстрой поперечной моды, $2-$ для медленной поперечной моды, 3 - для продольной моды, 4 - средняя длин свободного пробега, $5-$ средняя длина свободного пробега для полностью диффузного рассеяния. Точка - экспериментальное значение длины пробега для гетероструктур GaAs [1].

поскольку ось „1“ является осью вращения (см. формулы (5)-(6)).

Рассмотрим угловые зависимости длин свободного пробега в гетероструктуре GaAs с ориентацией $\{100\}$. Как видно из рис. 5, $a$, анизотропия теплопроводности и вкладов в нее от фононов различных поляризаций мала. В режиме кнудсеновского течения фононного газа она обусловлена медленной поперечной модой, которая фокусируется в направлении [100] и превышает значение в направлении дефокусировки [110] на 17\% (см. рис. 5, $a$ кривая 2). Анизотропия вклада быстрой 
поперечной моды мала: ее значение в направлении [110] на $3 \%$ превосходит величину в [100]. Анизотропия вклада продольных фононов также мала: ее значение в направлении [110] на 10\% превосходит величину в [100]. Поскольку максимумы вклада моды $t_{2}$ соответствуют минимумам вкладов быстрой поперечной моды и продольных фононов, то происходит частичная компенсация вкладов. В результате анизотропия полной теплопроводности гетероструктуры GaAs с ориентацией $\{110\}$ в режиме кнудсеновского течения фононного газа уменьшается до 7\% (см. рис. 5, $b$ кривая 5). Следует отметить, что мода $t_{2}$ вносит преобладающий вклад в теплопроводность гетероструктуры GaAs с ориентацией $\{100\}$ : он составляет 56, и 46\% для направлений [100] и [110] соответственно. Вклад продольных фононов существенно меньше: он составляет 8\% для направления [110], и уменьшается до 7\% для направления дефокусировки [100] (см. рис. 5, $a$ кривая 3). При полностью диффузном рассеянии фононов теплопроводность и средняя длина пробега в направлении [100] оказываются в 2.17 раза меньше экспериментального значения (см. рис. 4, $a$ штриховую 5).

Для второй гетероструктуры GaAs c ориентацией плоскости $\{110\}$ анизотропия теплопроводности значительно возрастает - она увеличивается в 5 раз. Максимум теплопроводности достигается в направлении [100] и оказывается на 35\% больше минимального значения в направлении [110]. Анизотропия теплопроводности в этом случае также обусловлена медленной поперечной модой, которая фокусируется в направлении [100] и дефокусируется в направлении в [110] (см. рис. 5, $b$ кривая 2). Отношение длин пробега для медленной поперечной моды в симметричных направлениях составляет $\Lambda_{[100]}^{\{110\} t_{2}}: \Lambda_{[111]}^{\{110\} t_{2}}: \Lambda_{[110]}^{\left\{110 t_{2}\right.}=1.9: 1.26: 1$. Вклад этой моды в теплопроводность гетероструктуры GaAs c ориентацией $\{110\}$ доминирует. Он составляет 66, 55 и 45\% для направлений [100], [110] и [111], соответственно. Анизотропия длин пробега для быстрой поперечной моды мала: для нее имеет вид $\Lambda_{[100]}^{\{110\} t_{1}}: \Lambda_{[110]}^{\{110\} t_{1}}: \Lambda_{[111]}^{\{110\} t_{1}} \cong 1.15: 1.1: 1$. Анизотропия длин пробега для продольных фононов значительно больше: отношение длин пробега для нее имеет вид $\Lambda_{[110]}^{\{110\} L}: \Lambda_{[111]}^{\{110\} L}: \Lambda_{[100]}^{\{110\} L}=1.71: 1.54: 1$ (см. рис. 5, $b$ кривая 3). Однако их вклад в теплопроводность мал: он достигает $21 \%$ для направления [111] и уменьшается для направления дефокусировки [100] до 7\%. Благодаря частичной компенсации вкладов анизотропия теплопроводности гетероструктуры GaAs c ориентацией $\{110\}$ уменьшается до $35 \%$ (см. рис. 5, $b$ кривая 5 ). Отношение средних длин пробега для нее имеет вид: $\Lambda_{[100]}^{\{110\}}: \Lambda_{[111]}^{\{110\}}: \Lambda_{[110]}^{\{110\}}=1.35: 1.08: 1$. Следует отметить, что при измерении теплопроводности гетероструктур с ориентациями плоскостей $\{100\}$ и $\{110\}$, имеющих одинаковые параметры зеркальности, для направлений теплового потока [100] и [110] мы получим: $\kappa_{[100]}^{\{100\}}: \kappa_{[100]}^{\{110\}}=1.2$ и $\kappa_{[110]}^{\{100\}}: \kappa_{[110]}^{\{110\}}=1.5$. Таким образом, для направления теплового потока [110] зависимость теплопроводности от ориентации гетероструктур оказывается заметно больше. Итак, в теплопроводности гетероструктур $\mathrm{GaAs} / \mathrm{AlGaAs}$ доминируют квазипоперечные фононы они обеспечивают от 80 до 93\% теплопереноса.

\section{4. Заключение}

Исследовано влияние фокусировки фононов на анизотропию теплопроводности гетероструктур GaAs/AlGaAs при низких температурах. Определены параметры зеркальности отражения фононов от границ гетероструктур, характеризующие тепловой поток в режиме кнудсеновского течения фононного газа. Рассчитаны угловые зависимости длин свободного пробега квазипродольных и квазипоперечных фононов, а также средних длин пробега, определяющих теплопроводность гетероструктур с ориентациями плоскостей $\{100\}$ и $\{110\}$. Показано, что теплопроводность гетероструктур с ориентациями плоскостей $\{100\}$ имеет малую анизотропию и значительно большие значения, чем для гетероструктур с ориентациями $\{110\}$. Изучена роль квазипродольных и квазипоперечных фононов в теплопроводности гетероструктур. Показано, что в доминирующий вклад вносят квазипоперечные фононы, которые обеспечивают от 80 до 93\% теплопереноса в гетероструктурах. Анизотропия теплопроводности гетероструктур в режиме кнудсеновского течения фононного газа обусловлена медленной поперечной модой, которая фокусируется в направлении [100] и обеспечивает максимальные значения теплопроводности.

\section{Список литературы}

[1] B. Tieke, R. Fletcher, U. Zeitler, M. Henini, J.C. Maan. Phys. Rev. B 58, 2017 (1998).

[2] R. Fletcher, J.C. Maan, G. Weimann. Phys. Rev. B 32, 8477 (1985).

[3] R. Fletcher, J.C. Maan, K. Ploog, G. Weimann. Phys. Rev. B 33, 7122 (1986).

[4] R. Fletcher, M. D'Iorio, A.S. Sachrajda, R. Stoner, C.T. Foxon, J.J. Harris. Phys. Rev. B 37, 3137(1988).

[5] D.G. Cantrell, P.N. Butcher. J. Phys. C 20, 1985 (1987).

[6] D.G. Cantrell, P.N. Butcher. J. Phys. C 20, 1993 (1987).

[7] D.G. Cantrell, P.N. Butcher. J. Phys. C 19, L429 (1986).

[8] H.J. Maris, S. Tamura. Phys. Rev. B 85, 054304 (2012).

[9] M.P. Zaitlin, L.M. Scherr, A.C. Anderson. Phys. Rev. B 12, 4487 (1975).

[10] B. Taylor, H.J. Maris, C. Elbaum. Phys. Rev. Lett. 23. 416 (1969).

[11] A.K. Mc Curdy, H.J. Maris, C. Elbaum. Phys. Rev. B 2, 4077 (1970).

[12] H.J.J. Maris. Acoust. Soc. Am. 50, 812 (1971).

[13] J.P. Wolfe. Imaging Phonons Acoustic Wave Propagation in Solids. Cambridge University Press, N.Y. (1998).

[14] И.И. Кулеев, И.Г. Кулеев, С.М. Бахарев, А.В. Инюшкин. ФTT 55, 24, (2013).

[15] I.I. Kuleyev, I.G. Kuleyev, S.M. Bakharev, A.V. Inyushkin. Physica B 416, 81 (2013). 
[16] I.I. Kuleyev, I.G. Kuleyev, S.M. Bakharev, A.V. Inyushkin. Phys. Status Solidi B 251, 991 (2014).

[17] И.Г. Кулеев, И.И. Кулеев, С.М. Бахарев, В.В. Устинов. Фокусировка фононов и фононный транспорт в монокристаллических наноструктурах. Изд-во УМЦ УПИ, Екатеринбург (2018). 256 с.

[18] Ф.И. Федоров. Теория упругих волн в кристаллах. Наука, M. (1965).

[19] B. Truel, C. Elbaum, B.B. Chick. Ultrasonic methods in sold state physics. Academic press, N. Y.-London (1969).

[20] И.Г. Кулеев, И.И. Кулеев. ФТТ 49, 422 (2007).

[21] J.R. Drabble, A.J. Brammer. Solid State Commun. 4, 467 (1966).

[22] J.S. Blakemore. J. Appl. Phys. 53, R123 (1982).

[23] I.I. Kuleyev, S.M. Bakharev, I.G. Kuleyev, V.V. Ustinov. Phys. Status Solidi C 14, 1600263 (2017).

[24] И.И. Кулеев, С.М. Бахарев, И.Г. Кулеев, В.В. Устинов. ФМM 118, 12 (2017).

[25] I.I. Kuleyev, I.G. Kuleyev, S.M. Bakharev. Phys. Status Solidi B 252, 323 (2015).

[26] Дж. Займан. Электроны и фононы. ИЛ, М. (1962).

[27] Б.М. Могилевский, А.Ф. Чудновский. Теплопроводность полупроводников. Наука, М. (1972). 536 с.

Редактор К.В. Емцев 